\title{
Energy Gap between Photoluminescence and Electroluminescence as Recombination Indicator in Organic Small-Molecule Photodiodes
}

Gae Hwang Lee, Moon Gyu Han*, Dong-Seok Leem, Seon-Jeong Lim, Sungyoung Yun, Kwang-Hee Lee, Xavier Bulliard, Kyung-Bae Park, Tadao Yagi, Yeong Suk Choi, Yong Wan Jin*, and Sangyoon Lee

Organic Materials Laboratory, Samsung Advanced Institute of Technology, Samsung Electronics, Co. Ltd, 130 Samsung-ro, Suwon-si, Gyeonggi-do 443-803, Republic of Korea

*To whom correspondence should be addressed, e-mail address: moongyu@samsung.com \& ywjin@samsung.com 


\section{Supporting Fig. S1, Lee et al.}

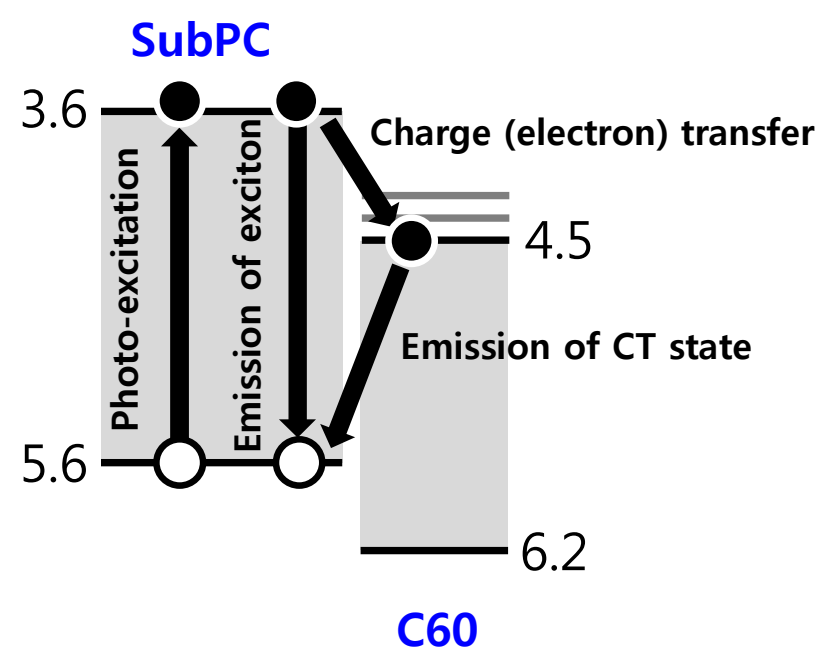

FIG. S1. A schematic diagram expressing the HOMO/LUMO energy level of SubPC and C60, excitation and emission procedures, and the charge transfer (CT) and emissive recombination procedures. Note that the CT state energy level of BHJ is constant when the p:n ratio is fixed but changes with the p:n ratios. 


\section{Supporting Fig. S2, Lee et al.}

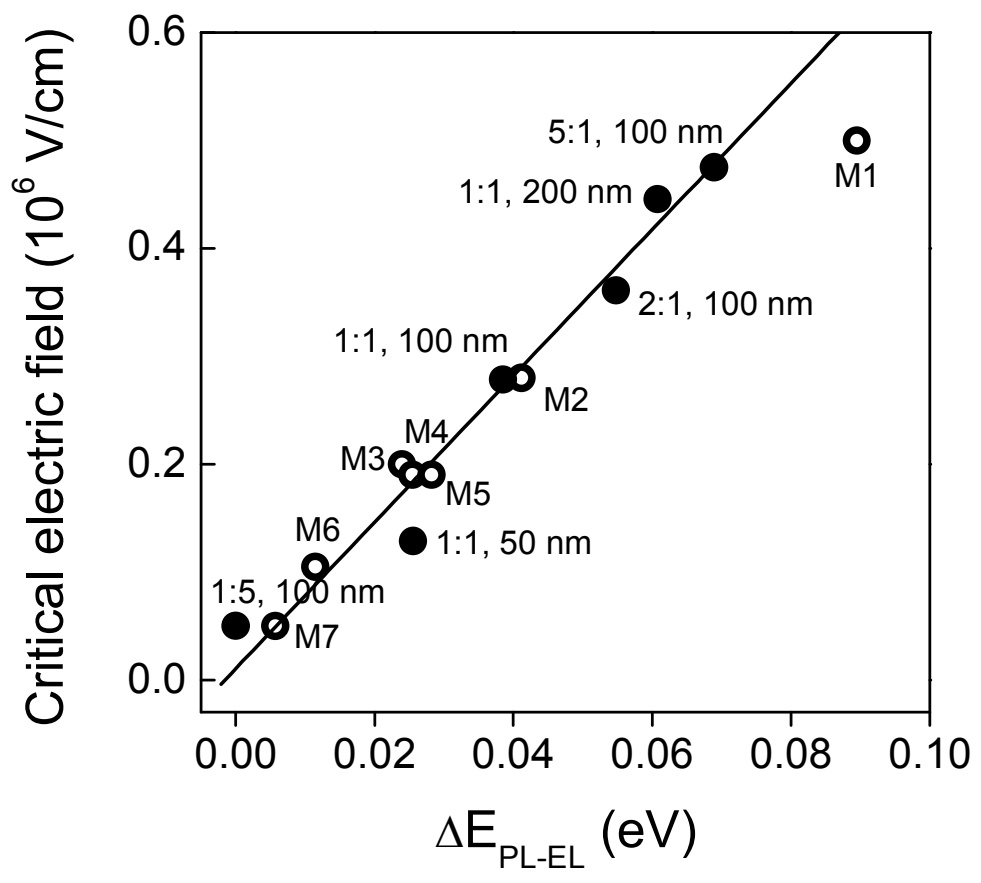

FIG. S2. Critical electric-field vs. energy gap in devices composed of C60 and different ptype molecules (The p-molecules are undisclosed yet). Newly added bulk heterojunctions are composed of 1:1 blend with thickness of $90 \mathrm{~nm}$ and are expressed in empty circle. M1-M4 are dipolar donor (D)-acceptor (A) molecules, M5 is Squaraine based D-A-D molecule, and M6-M7 are Boron-subphthalocyanine (SubPc) derivatives. The slop obtained from SubPc:C60 Blends remains valid even when the different p-type molecules were used in the bulk-heterojunction. 